\begin{tabular}{|c|c|c|c|c|}
\hline $\begin{array}{c}\text { Laboratory } \\
\text { number }\end{array}$ & $\begin{array}{l}\text { Published } \\
\text { reference }\end{array}$ & $\begin{array}{l}\text { Original date or } \\
\quad \text { other value }\end{array}$ & $\begin{array}{l}\text { Corrected date } \\
\text { or other value }\end{array}$ & $\partial \mathrm{C}^{14}, \%$ \\
\hline-757 & $166: 207$ & $3690 \pm 110$ & & \\
\hline-758 & $130: 121$ & $14,305 \pm 230$ & $14,305 \pm 230^{3}$ & \\
\hline-760 & $130: 126$ & $2460 \pm 110$ & & \\
\hline .770 & $130: 127$ & $5380 \pm 120$ & & \\
\hline-771 & $130: 131$ & $2460 \pm 200$ & & \\
\hline-771 & $130: 134$ & see Q-621 bis ${ }^{2}$ & & \\
\hline-790 & $166: 209$ & $7030 \pm 120$ & $7030 \pm 120^{3}$ & \\
\hline-791 & $166: 209$ & $5790 \pm 120$ & & \\
\hline-792 & $166: 209$ & $3928 \pm 110$ & $3928 \pm 100^{3}$ & \\
\hline .793 & $166: 210$ & $2428 \pm 110$ & $2428 \pm 110^{3}$ & \\
\hline-802 & $166: 212$ & $\partial \mathrm{C}^{14}+20 \%$ & & 20 \\
\hline-805 & $166: 210$ & $2495 \pm 110$ & & \\
\hline-806 & $166: 210$ & $2275 \pm 100$ & & \\
\hline-807 & $166: 210$ & $2377 \pm 100$ & & \\
\hline-810 & $166: 209$ & $4920 \pm 100$ & & \\
\hline-811 & $166: 209$ & $5530 \pm 100$ & & \\
\hline-815 & $166: 211$ & $10,300 \pm 185$ & & \\
\hline-818 & $166: 211$ & $6244 \pm 140$ & & \\
\hline-838 & $166: 211$ & $\Delta \mathrm{C}^{14} 767 \pm 20$ & & \\
\hline $\mathrm{QHF} / 62 / 0$ & $130: 135$ & $\partial \mathrm{C}^{14} \% 003529$ & & 3529 \\
\hline$-/ 62 / 1$ & $130: 135$ & $\partial \mathrm{C}^{14} \% 2000$ & & 2000 \\
\hline$-/ 62 / 2$ & $130: 135$ & $\partial \mathrm{C}^{14} \%$ & & 1723 \\
\hline$-/ 62 / 3$ & $130: 135$ & $\partial \mathrm{C}^{14} \%{ }_{00} 5882$ & & 5882 \\
\hline$-/ 62 / 4$ & $130: 135$ & $\partial \mathrm{C}^{14} \%{ }_{00} 2706$ & & 2706 \\
\hline$-/ 62 / 5$ & $130: 135$ & $\partial \mathrm{C}^{14} \%$ & & 1235 \\
\hline$-/ 62 / 6$ & $130: 135$ & $\partial \mathrm{C}^{14} \%{ }_{00} 2647$ & & 2647 \\
\hline$-/ 62 / 7$ & $130: 135$ & $\partial \mathrm{C}^{14} \%{ }_{00} 3765$ & & 3765 \\
\hline$-/ 62 / 8$ & $130: 135$ & $\partial \mathrm{C}^{14} \%{ }_{00} 1823$ & & 1823 \\
\hline$-/ 62 / 9$ & $130: 135$ & $\partial \mathrm{C}^{14} \%{ }_{00} 2588$ & & 2588 \\
\hline$-/ 62 / 10$ & $130: 135$ & $\partial \mathrm{C}^{14 \%} \% 1294$ & & 1294 \\
\hline$-/ 62 / 11$ & $130: 135$ & $\partial \mathrm{C}^{14} \%{ }_{00} 3470$ & & 3470 \\
\hline$-/ 62 / 12$ & $130: 135$ & $\partial \mathrm{C}^{14} \%{ }_{00} 1096$ & & 1096 \\
\hline$-/ 62 / 13$ & $130: 135$ & $\partial \mathrm{C}^{14} \% 1717$ & & 1717 \\
\hline$-/ 62 / 14$ & $130: 135$ & $\partial \mathrm{C}^{14} \%{ }_{00} 877$ & & 877 \\
\hline$-/ 62 / 15 \mathrm{~A}$ & $130: 135$ & $\partial \mathrm{C}^{14} \%{ }_{00} 650$ & & 650 \\
\hline$. / 62 / 15 \mathrm{~B}$ & $130: 135$ & $\partial \mathrm{C}^{14} \%{ }_{00} 1055$ & & 1055 \\
\hline$-/ 62 / 16 \mathrm{~A}$ & $130: 135$ & $\partial \mathrm{C}^{14} \% 1823$ & & 1823 \\
\hline$-/ 62 / 16 \mathrm{~B}$ & $130: 135$ & $\partial \mathrm{C}^{14} \%{ }_{00} 2754$ & & 2754 \\
\hline$-/ 63 / 17$ & $130: 135$ & $\partial \mathrm{C}^{14} \%{ }_{00} 3690$ & & 3690 \\
\hline$-; 63 / 18 A$ & $130: 135$ & $\partial \mathrm{C}^{1.4} \%{ }_{00} 9022$ & & 9022 \\
\hline$-/ 63 / 18 B$ & $130: 135$ & $\partial \mathrm{C}^{14} \%{ }_{00} 5377$ & & 5377 \\
\hline$-/ 63 / 19$ & $130: 135$ & $\partial \mathrm{C}^{14} \% 5854$ & & 5854 \\
\hline$-/ 63 / 20$ & $130: 135$ & $\partial \mathrm{C}^{14} \%{ }_{00} 4804$ & & 4804 \\
\hline$-/ 63 / 21$ & $130: 135$ & $\partial \mathrm{C}^{14} \%{ }_{00} 5386$ & & 5386 \\
\hline
\end{tabular}

${ }^{1}$ Reference standard for earlier samples was age-corrected pre-industrial wood, shown (by Q-462) not to differ significantly from $0.95 \times$ NBS oxalic acid standard.

${ }^{2}$ Pub. as Q-771, ref. 130, p. 134, rec p. 131.

${ }^{3}$ Original duplicate measurements have been averaged arithmetically.

\title{
University of Rome, Carbon-14 Dating Laboratory
}

$\begin{array}{clccc}\begin{array}{c}\text { Laboratory } \\ \text { number }\end{array} & \begin{array}{c}\text { Published } \\ \text { reference }\end{array} & \begin{array}{c}\text { Original date or } \\ \text { other value }\end{array} & \begin{array}{c}\text { Corrected date } \\ \text { or other value }\end{array} & \text { oth } \mathrm{C}^{14}, \% 0 \\ \mathrm{R}-1^{2} & 97 \mathrm{a}: 2679 & 2125 \pm 75^{\mathrm{s}} & & \\ -1^{2} & 125: 78 & 1990 \pm 85 & & \\ -2 & 97 \mathrm{a}: 2679 & 1810 \pm 140^{\mathrm{s}} & \\ -3 & 125: 82 & 1900 \pm 80 & \\ -4 & 97 \mathrm{a}: 2679 & 1650 \pm 180^{\mathrm{s}} & \\ -5 & 167: 216 & 700 \pm 60 & \\ -6 & 97 \mathrm{a}: 2679 & 2730 \pm 240^{\mathrm{s}} & \mathrm{d}\end{array}$

A.D./ B.C. date 175 B.C. 40 B.C.

A.D. 140

A.D. 50

A.D. 300

A.D. 1250 


\begin{tabular}{|c|c|c|c|c|}
\hline $\begin{array}{l}\text { Laboratory } \\
\text { number }\end{array}$ & $\begin{array}{l}\text { Published } \\
\text { reference }\end{array}$ & $\begin{array}{l}\text { Original date or } \\
\quad \text { other value }\end{array}$ & $\begin{array}{l}\text { Corrected date } \\
\text { or other value }\end{array}$ & $\partial \mathrm{C}^{14}, \%$ \\
\hline-6 & $167: 216$ & $2380 \pm 60$ & & \\
\hline-7 & $125: 82$ & $3310 \pm 210$ & & \\
\hline-9 & $125: 81$ & $2460 \pm 100$ & $d$ & \\
\hline-9 & $167: 217$ & $2870 \pm 60$ & & \\
\hline .10 & $125: 82$ & $2140 \pm 65$ & & \\
\hline$-10 \mathrm{~A}$ & $167: 216$ & $2175 \pm 45$ & & \\
\hline$-10 \mathrm{~B}$ & $167: 216$ & $2250 \pm 45$ & & \\
\hline-11 & $125: 87$ & $8000 \pm 100$ & & \\
\hline-12 & $125: 87$ & $6400 \pm 200$ & & \\
\hline-13 & $125: 87$ & $5550 \pm 70$ & & \\
\hline-14 & $125: 87$ & $4660 \pm 80$ & & \\
\hline .15 & $125: 87$ & $3000 \pm 100$ & & \\
\hline-17 & $125: 83$ & $2120 \pm 190$ & & \\
\hline .19 & $125: 83$ & $2600 \pm 150$ & & \\
\hline-20 & $125: 83$ & $2280 \pm 250$ & & \\
\hline-21 & $125: 83$ & $2360 \pm 85$ & & \\
\hline$-22 \mathrm{~A}$ & $125: 83$ & $2280 \pm 90$ & & \\
\hline$-22 \mathrm{~B}$ & $125: 83$ & $2260 \pm 80$ & & \\
\hline-23 & $125: 84$ & $280 \pm 80$ & & \\
\hline-24 & $167: 217$ & $2700 \pm 60$ & & \\
\hline-25 & $167: 215$ & $3495 \pm 60$ & & \\
\hline-26 & $167: 216$ & $1935 \pm 55$ & & \\
\hline-27 & $167: 213$ & $2190 \pm 65$ & & \\
\hline-28 & $167: 218$ & $2350 \pm 65$ & & \\
\hline$-29 \mathrm{~A}$ & $125: 81$ & $580 \pm 50$ & & \\
\hline-32 & $167: 217$ & $2515 \pm 45$ & & \\
\hline-33 & $167: 219$ & $3300 \pm 40$ & & \\
\hline-35 & $125: 84$ & $3880 \pm 80$ & & \\
\hline-36 & $167: 219$ & $2950 \pm 40$ & & \\
\hline-37 & $125: 84$ & $2410 \pm 50$ & & \\
\hline-38 & $97 a: 2679$ & $3600 \pm 700^{\mathrm{s}}$ & & \\
\hline-38 & $167: 219$ & $3200 \pm 45$ & & \\
\hline-39 & $167: 220$ & $18.550 \pm 400$ & & \\
\hline-39 & $125: 87$ & $18,800 \pm 400$ & & \\
\hline .40 & $125: 88$ & $4400 \pm 300$ & & \\
\hline-41 & $125: 88$ & $2950 \pm 250$ & & \\
\hline-42 & $125: 88$ & $4200 \pm 300$ & & \\
\hline .43 & $125: 88$ & $5600 \pm 300$ & & \\
\hline-44 & $125: 88$ & Not dated & & \\
\hline-45 & $125: 88$ & $1300 \pm 250$ & & \\
\hline-46 & $125: 88$ & $1750 \pm 250$ & & \\
\hline-47 & $125: 88$ & $2400 \pm 300$ & & \\
\hline-48 & $125: 88$ & $4800 \pm 300$ & & \\
\hline-49 & $125: 88$ & $2400 \pm 250$ & & \\
\hline-50 & $125: 88$ & $2750 \pm 250$ & & \\
\hline-51 & $125: 88$ & $2850 \pm 250$ & & \\
\hline-52 & $125: 86$ & $7780 \pm 100$ & & \\
\hline-53 & $125: 86$ & $2020 \pm 100$ & & \\
\hline-54 & $167: 218$ & $9050 \pm 100$ & & \\
\hline$-56^{5}$ & -:94 & $11,930 \pm 520$ & & \\
\hline-58 & $125: 79$ & $11,800 \pm 600$ & & \\
\hline-63 & $125: 82$ & $340 \pm 60$ & & \\
\hline$-64^{3}$ & $125: 78$ & $11,900 \pm 170$ & $\mathrm{~d}$ & \\
\hline$-64^{4}$ & $167: 220$ & $11,200 \pm 145$ & & \\
\hline-66 & $125: 81$ & $3500 \pm 250$ & & \\
\hline-78 & $125: 81$ & $4200 \pm 300$ & & \\
\hline-80 & $167: 215$ & $470 \pm 45$ & & \\
\hline .81 & $125: 86$ & $3200 \pm 75$ & & \\
\hline-82 & $125: 86$ & $3600 \pm 60$ & & \\
\hline-83 & $125: 79$ & $13,000 \pm 700$ & & \\
\hline-84 & $125: 85$ & $4.30 \pm 80$ & & \\
\hline
\end{tabular}

A.D./

B.C. date

430 B.C.

1360 B.C.

920 B.C.

190 B.C.

225 B.C.

300 B.C.

6050 B.C.

4450 B.C.

3600 B.C.

2710 B.C.

1050 B.C.

170 B.C.

650 B.C.

330 B.C.

410 B.C.

330 B.C.

310 B.C.

A.D. 1670

750 B.C.

1545 B.C.

A.D. 15

240 B.C.

400 B.C.

A.D. 1370

565 B.C.

1350 B.C.

1930 B.C.

1000 B.C.

460 B.C.

1650 B.C.

1250 B.C.

16,600 B.C.

16,850 B.C.

2450 B.C.

1000 B.C.

2250 B.C.

3650 B.C.

A.D. 650

A.D. 200

450 B.C.

2850 B.C.

450 B.C.

800 B.C.

900 B.C.

5830 B.C.

70 B.C.

7100 B.C.

9980 B.C.

9850 B.C.

A.D. 1610

9950 B.C.

9250 B.C.

1550 B.C.

2250 B.C.

A.D. 1480

1250 B.C.

1650 B.C.

11,050 B.C.

A.D. 1520 


$\begin{array}{cccc}\begin{array}{c}\text { Laboratory } \\ \text { number }\end{array} & \begin{array}{c}\text { Published } \\ \text { reference }\end{array} & \begin{array}{c}\text { Original date or } \\ \text { other value }\end{array} & \begin{array}{c}\text { Corrected date } \\ \text { or other value }\end{array} \\ -85 & 125: 85 & 430 \pm 90 & \\ -86 & 125: 85 & 370 \pm 80 & \\ -92 \mathrm{~A} & 125: 82 & 1890 \pm 90 & \\ -92 \mathrm{~B} & 125: 82 & 1850 \pm 85 & \\ -93 & 167: 218 & 200 \pm 200 & \\ -94 \mathrm{~A} & 167: 214 & 2050 \pm 50 & \\ -94 \mathrm{~B} & 167: 214 & 2440 \pm 55 & \\ -95 & 167: 213 & 5825 \pm 75 & \\ -96 & 167: 214 & 3230 \pm 60 & \\ -97 & 167: 215 & 3475 \pm 60 & \\ -98 & 167: 215 & 3075 \pm 60 & \\ -106^{4} & 125: 79 & 11,800 \pm 280 & \\ -109 & 167: 215 & 7800 \pm 100 & \\ -113 & 125: 80 & 2100 \pm 80 & \\ -114 & 125: 80 & 2970 \pm 90 & \\ -117 & 167: 217 & 3100 \pm 60 & \\ -122 & 125: 81 & 4650 \pm 90 & \\ -130 & 167: 218 & 13,170 \pm 175 & \end{array}$

A.D./

B.C. date

A.D. 1520

A.D. 1580

A.D. 60

A.D. 100

A.D. 1750

100 B.C.

490 B.C.

3875 B.C

1280 B.C.

1525 B.C.

1125 B.C.

9850 B.C.

5850 B.C

150 B.C.

1020 B.C.

1150 B.C.

2700 B.C.

11,220 B.C.

${ }^{1}$ Reference standard: Wood grown near Rome between 1949 and 1953.

${ }^{2}$ Cross-check sample (R-1) distributed by Rome Lab. to many laboratories: T-9; St103/A/B； BM-15; Q-112; U-68

${ }^{3}$ R-64, cross-check sample distributed by H. Tauber, Copenhagen Lab., (K-101) to many labs.: K-101 bis; K-102 bis; Gro-454; St-18; U-20; U-75; H-105; BM-19; W-82, W-84.

${ }^{4}$ Sample given us by Hl. de Vries at Groningen (Nov. 1957); it is the sample pub. as Gro-933 and Gro-948.

${ }^{5}$-Pub. in Quaternaria, v. 5, p. 94.

\section{University of Saskatchewan, Department of Chemistry}

\begin{tabular}{|c|c|c|c|c|c|}
\hline $\begin{array}{l}\text { Laboratory } \\
\text { number }\end{array}$ & $\begin{array}{l}\text { Published } \\
\text { reference }\end{array}$ & $\begin{array}{l}\text { Original date or } \\
\text { other value }\end{array}$ & $\begin{array}{l}\text { Corrected date } \\
\text { or other value }\end{array}$ & $\partial \mathrm{C}^{14}, \%$ & $\begin{array}{l}\text { A.D./ } \\
\text { B.C. date }\end{array}$ \\
\hline S-1 & 180 & $1910 \pm 130^{\mathrm{s}}$ & & $211 \pm 17$ & A.D. 40 \\
\hline-2 & 180 & $3400 \pm 200^{s}$ & $2780 \pm 200 \mathrm{c}$ & $292 \pm 16$ & 830 B.C. \\
\hline-3 & 180 & $2430 \pm 160^{\mathrm{s}}$ & & $260 \pm 16$ & 480 B.C. \\
\hline-4 & 180 & $2415 \pm 160^{\mathrm{s}}$ & & $268 \pm 16$ & 465 B.C. \\
\hline-7 & 180 & $>25,000^{\mathrm{s}}$ & & $966 \pm 16$ & \\
\hline-12 & 180 & $3670 \pm 270^{s}$ & & $367 \pm 21$ & 1720 B.C. \\
\hline-13 & 180 & $5600 \pm 300^{s}$ & & $502 \pm 21$ & 3650 B.C. \\
\hline-16 & $89: 73$ & $6250 \pm 250$ & $6300 \pm 250 \mathrm{a}$ & $544 \pm 11$ & 4350 B.C. \\
\hline .17 & 180 & d & see $\mathrm{S}-17$ bis & & \\
\hline-17 bis & $89: 77$ & $2350 \pm 60$ & & $252 \pm 6$ & 400 B.C. \\
\hline-18 & $89: 77$ & $2450 \pm 160$ & & $264 \pm 11$ & 500 B.C. \\
\hline-19 & $89: 77$ & $1580 \pm 140$ & & $179 \pm 12$ & A.D. 370 \\
\hline-20 & $89: 77$ & $660 \pm 130$ & & $80 \pm 10$ & A.D. 1290 \\
\hline-22 & 180 & $1580 \pm 159^{s}$ & & $179 \pm 24$ & A.D. 370 \\
\hline-25 & 180 & $4400 \pm 216^{s}$ & d & $438 \pm 23$ & \\
\hline$-25^{1}$ & $89: 74$ & $4600 \pm 210$ & $4650 \pm 210 \mathrm{a}$ & $439 \pm 15$ & 2700 B.C. \\
\hline-26 & 180 & $>23,000^{\mathrm{s}}$ & & $1015 \pm 28$ & \\
\hline-28 & 180 & $2400 \pm 173^{\mathrm{s}}$ & & $258 \pm 24$ & 450 B.C. \\
\hline-29 & $89: 74$ & $11,400 \pm 450$ & & $760 \pm 11$ & 9450 B.C. \\
\hline-30 & $89: 74$ & $12,000 \pm 500$ & & $777 \pm 11$ & 10,050 B.C. \\
\hline-31 & 180 & $12,660 \pm 440^{\mathrm{s}}$ & & $793 \pm 21$ & 10,710 B.C. \\
\hline-32 & $90: 71$ & $7800 \pm 300$ & $7850 \pm 300 \mathrm{a}$ & $622 \pm 11$ & 5900 B.C. \\
\hline-33 & $90: 71$ & $2980 \pm 175$ & $3030 \pm 175$ a & $313 \pm 11$ & 1080 B.C. \\
\hline-34 & $90: 71$ & $8180 \pm 320$ & $8230 \pm 320$ a & $670 \pm 12$ & 6280 B.C. \\
\hline-40 & $89: 76$ & $>33,000$ & & VJJD $\pm v C$ & \\
\hline-41 & $89: 74$ & $10,000 \pm 300$ & & $710 \pm 13$ & 8050 B.C. \\
\hline-42 & $89: 76$ & $>30,000$ & & $992 \pm 13$ & \\
\hline-43 & $89: 79$ & $400 \pm 120$ & $450 \pm 120 \mathrm{a}$ & $43 \pm 11$ & A.D. 1600 \\
\hline
\end{tabular}

